\title{
Analysing and Inferring of Intimacy Based on fNIRS Signals and Peripheral Physiological Signals
}

\author{
Chao Li*, Qian Zhang*, Ziping Zhao*, Li Gu ${ }^{\dagger}$, Nicholas Cummins ${ }^{\ddagger}$, Björn Schuller ${ }^{\ddagger} \S$ \\ ${ }^{*}$ College of Computer and Information Engineering, Tianjin Normal University, Tianjin, China, ztianjin@126.com \\ ${ }^{\dagger}$ Institute of psychology and behavior, Tianjin Normal University, Tianjin, China \\ ${ }^{\ddagger}$ ZD.B Chair of Embedded Intelligence for Health Care and Wellbeing, University of Augsburg, Augsburg, Germany \\ $\S$ Group on Language, Audio \& Music, Imperial College London, London, UK
}

\begin{abstract}
Intimacy refers to a relatively long-lasting affinity relationship between individuals, which involves complex neuronal activities and physiological changes in the body. Recent advancements in the field of neuroimaging have demonstrated that functional near-infrared spectroscopy (fNIRS) has excellent potential for intimate relationship analysis. Signals such as fNIRS and physiological signals are increasingly utilised in this regard due to their consistency and complementarity. In this paper, first, we apply fNIRS and physiological database collected from 26 subjects when viewing lover, friend and stranger pictures to analyse and infer the intimacy. Then, the time domain information from both the fNIRS and physiological signals are utilised to exploit the representation of intimacy by General Linear Model (GLM) and Complex Brain Network Analysis (CBNA) methods. Based on these two methods, the intimacy can be analysed with different brain activation patterns. Finally, different machine learning techniques are utilised to predict the intimate relationship. The results demonstrate that multi-modal features are more efficient for intimacy research. Moreover, the average classification accuracy of ensemble learning is $98.72 \%$ whereas for $\mathrm{KNN}$ it is $\mathbf{9 1 . 0 3 \%}$.

Index Terms-Intimacy, Functional near-infrared spectroscopy signals, Physiological signals, General Linear Model, Complex Brain Network Analysis
\end{abstract}

\section{INTRODUCTION}

Intimacy generally refers to the feeling of being in a close personal association and belonging together. It plays an essential role in our lives. Intimacy emphasises the degree of interdependence between the two sides [1], which can be the romantic relationship of lovers, a marriage relationship between husband and wife, or an intimate friendship. Whether it is to build a happy family or a happy organisation, an intimate relationship is important.

Functional magnetic resonance imaging (fMRI) has been widely used to analyse intimate relationship, for example in [2] [3]. However, the utilisation of fMRI has some limitations mainly due to the large size and the high cost of scanners. Recently, functional near-infrared spectroscopy (fNIRS) [4] [5] [6] has been performed in the field of laboratory advanced

The work presented in this paper was substantially supported by the National Natural Science Foundation of China (Grant No: 61702370), the Key Program of the Natural Science Foundation of Tianjin (Grant No. 18JCZDJC36300), the Open Projects Program of the National Laboratory of Pattern Recognition and the Senior Visiting Scholar Program of Tianjin Normal University.Chao Li and Qian Zhang share joint first authorship. Ziping Zhao is the corresponding author. cognitive neuroscience research [7] [8], brain-computer interface research [9] [10], and other cognitive activities [11] [12] [13]. It has the advantages of low cost, good portability, noninvasiveness and no excessive sensitivity to the test activities during the experiment. Therefore, researchers are increasingly choosing fNIRS as a means of data collection. The complementary principle of multi-modal information states that each modality contains some knowledge that the other modalities do not have. Signals produced by fNIRS can reflect the complex neuronal activities in the body. Physiological signals, on the other hand, reflect the physical changes on the autonomic nervous system. Physiological signals can direct reflect physical reactions that the human body produces, easy to collect by wearable devices, and hard to be affected by external environment [14] [15]. Therefore, the instinctive idea is to fuse two modalities to analyse intimate relationship.

Based on the above considerations, we propose a complete framework for analysing and inferring the intimate relationship using fNIRS and physiological signals. The framework has five key steps (Fig. 1). The first step is signal collection and noise removal - collecting fNIRS and physiological signals, then removing noise from raw data using a bandpass filtering method. The second step is then feature extraction - the time domain information from both fNIRS and physiological signals are extracted. The third step is Feature fusion and feature selection - fusing features by the z-score method, then comparing two different feature selection techniques between linear discriminant analysis (LDA) and principal component analysis (PCA) to select more effective features. The fourth step is model training - dividing the selected feature set into a training set and a test set, machine learning techniques are then utilised to predict the model performance. The final step is calculating the cclassification accuracy - outputting the results using different classifiers.

Furthermore, passion, as the most changeable and least controllable component of love, is critical in the study of intimacy. Passion is also highly related to sensitivity [16]. The construct of sensitivity can be divided into Sensitivity to Reward (SR), and Sensitivity to Punishment (SP), where SR reflects the responsiveness of people when presenting reward signals or withdrawing punishment signals, and SP the responsiveness of people when they present a penalty signal or withdraw the reward signal [17]. It has been shown that 


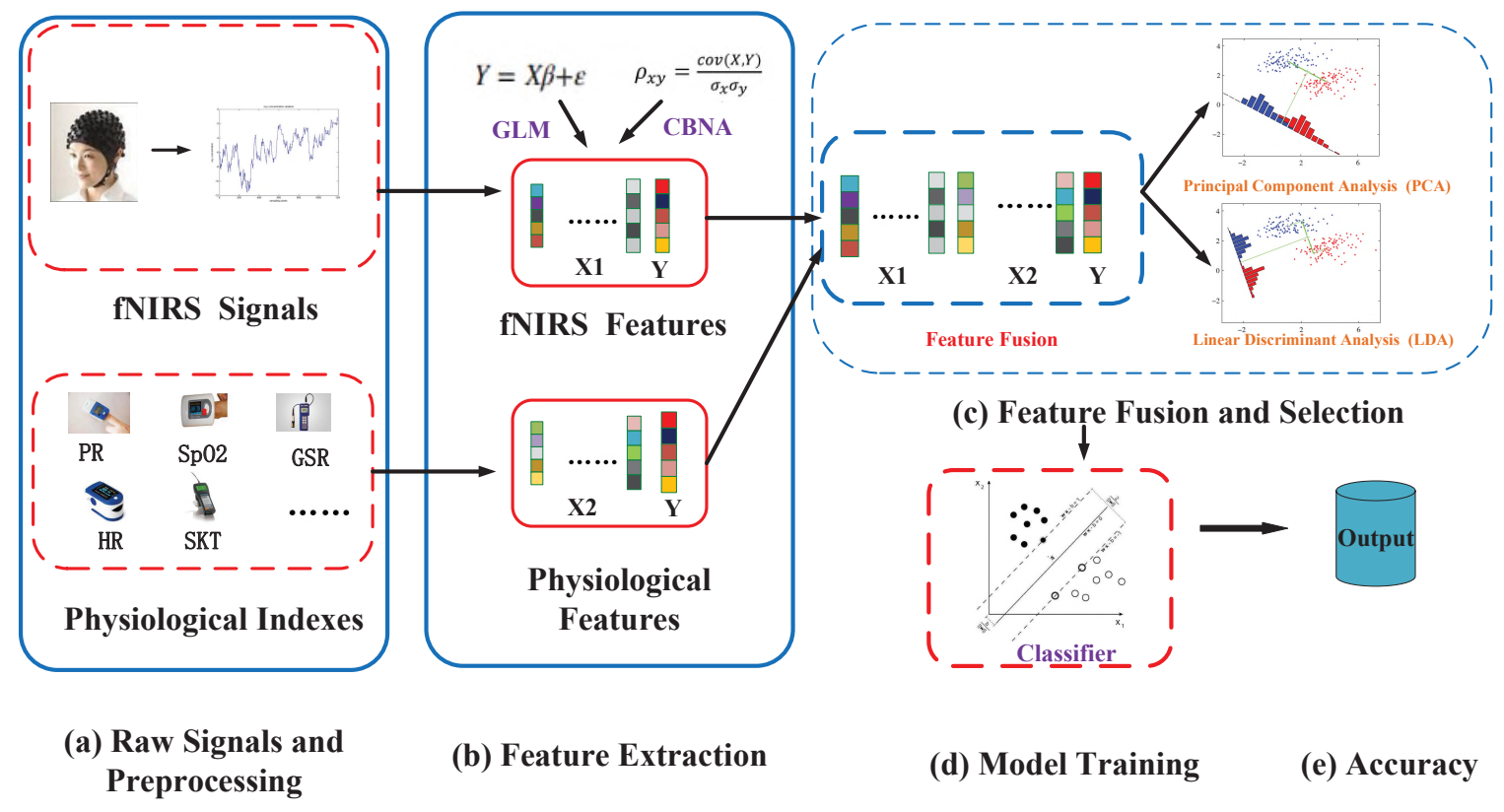

Fig. 1. The basic framework of inferring intimacy based on fNIRS signals and physiological signals

the SR in the intensified sensitivity is more closely related to the self-reported passion of the individual, while the SP is not significantly related to the passion [16]. This study, however, did not further predict the relationship. Based on this, we herein use different SR levels to analyse the activation patterns of different intimate relationships and make predictions.

Our contributions can, therefore, be summarised as follows:

- The brain activation patterns of fNIRS signals are analysed using General Linear Models (GLM), with our presented findings consistent with previous studies [16]. Moreover, a brain function network of fNIRS signals is constructed, which confirms that the brain network has small-world characteristics. Based on this network topology, the correlation between brain regions is revealed from a global perspective.

- SR has a positive predictive effect on the passion of intimacy. We analyse the intimacy based on the brain activation degree affected by different SR levels. The results indicate that individuals with a mean range SR level are easier to predict with respect to the analysis of intimate relationships. The average is more comprehensive and intuitive to consider for the characteristics of each data.

The remainder of this paper is organised as follows: Section II introduces the basic GLM and Complex Brain Network Analysis (CBNA) algorithms. Section III introduces the experimental setting, including a description of the database, data preprocessing, the feature extraction, and classification using machine learning methods. Section IV analyses the experimental results. Section V summarises this paper and outlines the future work.

\section{METHOD}

In this section, we introduce our methods for analysing and inferring intimate relationships based on fNIRS and physiological signals.

\section{A. General Linear Model}

The GLM algorithm has developed into the most commonly used method for estimating brain activation intensity in fNIRS data analysis and is often utilised in the selection of Regions of Interest (ROI) [18] [19]. GLM measures the temporal variational pattern of signals, rather than their absolute magnitude. GLM is known to be robust against common fNIRS confounders such as cases with an incorrect diffusion pathlength factor (DPF) or cases with severe optical signal attenuation due to scattering or weak contact. GLM effectively reduces the influence of error or non-task variables and can obtain activation parameters under different experimental conditions in the same model. These characteristics mean GLM is more convenient for comparative analysis of brain activities under different experimental conditions at the whole group level [20].

The GLM algorithm [21] expresses the observed response $(\mathrm{Y})$ as a linear combination of explanatory variables (X) plus an error term $(\varepsilon)$ :

$$
Y=x * \beta+\varepsilon,
$$

where, $\mathrm{Y}$ is an $\mathrm{N}^{*} \mathrm{M}$ matrix representing the measured fNIRS signals, in which $\mathrm{N}$ is the number of time points and $\mathrm{M}$ is the number of channels; $\mathrm{X}$ is an $\mathrm{N} * \mathrm{~W}$ design matrix composed by W-regressors. Regressors are computed by convolving a boxcar function with the canonical hemodynamic response function (HRF). $\beta$ is an $\mathrm{W}^{*} \mathrm{M}$ regression coefficients matrix, which indicates the contribution of each regressor to the observed responses $(\mathrm{Y})$, the greater the value representing, the 


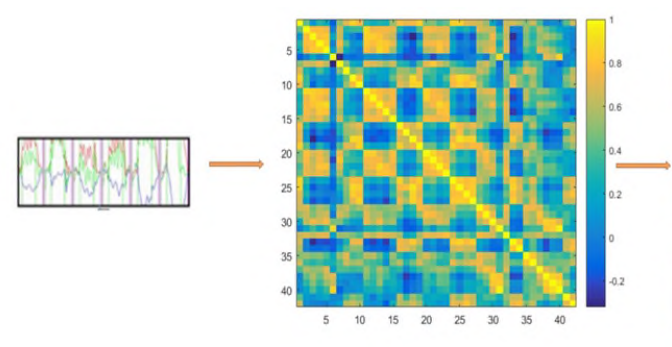

(a) fNIRS Time Series (b) Correlation Matrix

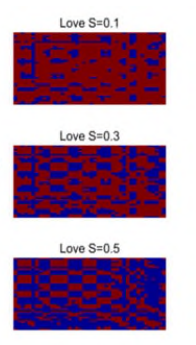

(c) Binary Matrix

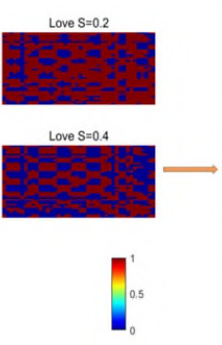

.

.

Fig. 2. Flow chart of brain network function connection

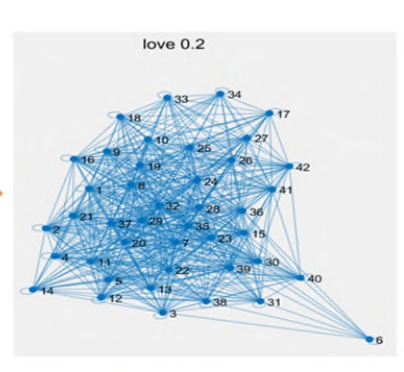

(d) Brain Network Diagram higher the activation of the brain under this condition. Finally, $\varepsilon$ is an $\mathrm{N}^{*} \mathrm{M}$ error matrix which includes the independent and identically distributed errors, with 0 mean and $\sigma^{2}$ variance $\left(\varepsilon \sim N\left(0, \sigma^{2} I\right)\right.$ ), representing the residual variance in the observed responses not explained by the model. Moreover, it is necessary to evaluate the effect of the experiment, to determine whether the expected result can be achieved, and to provide statistical guidance for the selection of the classification feature.

\section{B. Brain function connection analysis}

The human brain is a complex and efficient network system; therefore, a cognitive task usually requires the participation of multiple functional areas [22]. GLM is modelled independently for each channel, and cannot estimate the activation patterns of multiple regions of the brain. Therefore, in addition to detecting activated regions, it is also an ongoing challenge in brain function research to study how these brain regions are connected to form a dynamic functional network [23].

In the brain network analysis method, the commonly used seed-based correlation analysis (SCA) is limited, in that only two variables can be calculated separately. Differing from SCA, small world network analysis, a graph-based analysis method, offers the advantage in that the whole brain can be regarded in the analysis. This advantage is based on network topology research which reveals the correlation of brain intervals from a global perspective [24].

Construction of the brain network: With the centre of each channel as the node, the connection strength between the nodes is expressed by the correlation of the measurement results. The Pearson correlation coefficient is typically used to measure the connection between the fNIRS channels.

The Pearson correlation coefficient refers to the correlation between two variables $\mathrm{X}$ and $\mathrm{Y}$ (linear correlation); its value is between -1 and 1 [12]. The Pearson correlation coefficient between two variables is defined as the quotient of the covariance and standard deviation between the two variables. In this experiment, the variables $\mathrm{X}, \mathrm{Y}$ refer to the $\mathrm{i}$-th and $\mathrm{j}$-th channel data $(\mathrm{i}, \mathrm{j}=1,2,3, \ldots 42, i \neq j)$. It is expressed by $\rho_{i, j}$ :

$$
\begin{aligned}
\rho_{i, j} & =\frac{\operatorname{COV}(X, Y)}{\sigma_{X} \sigma_{Y}}, \\
& =\frac{E\left(X-\mu_{X}\right)\left(Y-\mu_{Y}\right)}{\sigma_{X} \sigma_{Y}}, \\
& =\frac{E(X Y)-E(X) E(Y)}{\sqrt{E\left(X^{2}\right)-E^{2}(X)} \sqrt{E\left(Y^{2}\right)-E^{2}(Y)}},
\end{aligned}
$$

where $\operatorname{cov}(\mathrm{X}, \mathrm{Y})$ is the covariance, $\sigma_{X}, \sigma_{Y}$ are the standard deviation, $\mu_{X}, \mu_{Y}$ are the mean of variable $\mathrm{X}$ and $\mathrm{Y}$ respectively.

Choice of brain network threshold: The weight matrix $\mathrm{W}$ of the network can be obtained by calculating the correlation between the two nodes. In brain network analysis, the adjacency matrix with weaker connections is usually utilised to analyse the characteristics of the network. Therefore, it is necessary to set a sparsity threshold to convert the weight matrix into a binary adjacency matrix, that is, a connection coefficient greater than the threshold is set to 1 , and smaller than the threshold is set to 0 .

The brain network can be considered a sparse network when the sparsity threshold is less than 0.5 ; furthermore, the network efficiency presents a nonlinear overshoot as the sparsity increases. It is also important to note that when the sparsity threshold is greater than 0.5 , the brain network degenerates into a random network. Therefore, we have to select the appropriate sparsity range under the premise of ensuring the small world attribute of the brain network and to adequately characterise the network in this range space. Typically, the sparsity threshold space is set to range from 0.1 to 0.5 with an interval of 0.1 . The flow chart of brain network construction is shown in Fig. 2.

\section{EXPERIMENTAL DESIGN}

This section outlines all key settings used in our experimental analysis.

\section{A. Database}

In order to demonstrate that our methods can effectively analyse and infer intimacy, we validate them based on a database provided by the Institute of Psychology and Behaviour of Tianjin Normal University [16]. This corpus includes the 


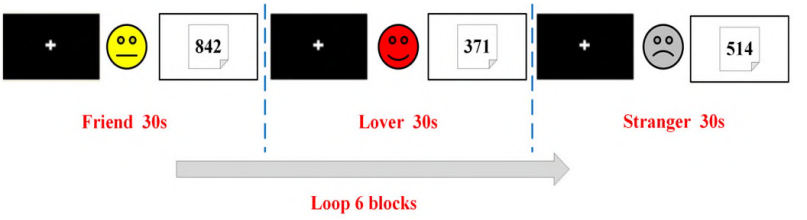

Fig. 3. Experimental paradigm for inducing subject's fNIRS and physiological response with different intimate relationships

fNIRS and the physiological signals sets. An overview of the experimental paradigm associated with the database is shown in Fig. 3. In total, fNIRS and physiological signals of 26 subjects were recorded while they were watching three different types of pictures: friends, lovers, and strangers. The recorded physiological signals mainly include the blood oxygen saturation $(\mathrm{SpO} 2)$, heart rate $(\mathrm{HR})$, galvanic skin response (GSR), skin temperature (SKT), and pulse rate (PR).

A random number (such as 842 ) was presented in the gap between pictures, with the subject being asked to cycle minus 7 until the number disappears. The purpose of this step is to allow the subject adequate time to, cognitively, eliminate the emotional stimulation after viewing the different pictures, and restore their neurophysiological baseline. The timing of the random number presentations is: 20 s after the picture of the friend and stranger, and 40s after the picture of the lover. The reason why the time of lovers is longer after the lover's picture was presented is that the lover's stimulation as a high wakeup stimulus requires more time to eliminate the emotional excitement

Changes in cerebral cortex oxyhemoglobin concentration $\left(\mathrm{HbO}_{2}\right)$ are analysed via the fNIRS images. The sampling rate of the instrument used in the experiment was $11 \mathrm{~Hz}$. For the physiological database, each physiological index measured is averaged and subtracted from the baseline value at each stage of the experiment, and the results obtained are changes in these physiological indicators.

\section{B. Preprocessing}

When acquiring signals using fNIRS technology, noise is often generated through the subject's head movements and heartbeat, as well as instrument interference, and the like. In order to obtain more accurate and reasonable data, we need to process the data first. There are many possible methods for preprocessing fNIRS signals, such as Hilbert Huang transform, band-pass filtering, Fourier spectrum analysis, and multiresolution analysis of wavelet transaction. In this experiment, the frequency range of the fNIRS signals is determined by Fourier spectrum analysis to be $0.01-0.2 \mathrm{~Hz}$. The filtering result of the first subject viewing a lover's picture using the band-pass filter is given in Fig. 4, the blue line represents the original data, and the red line represents the data after bandpass filtering. It can be seen in this exemplary image that after band-pass filtering, the signal is smoother, and that the high-frequency noise is reduced. Initial experiments indicated that these effects improved the classification accuracy.

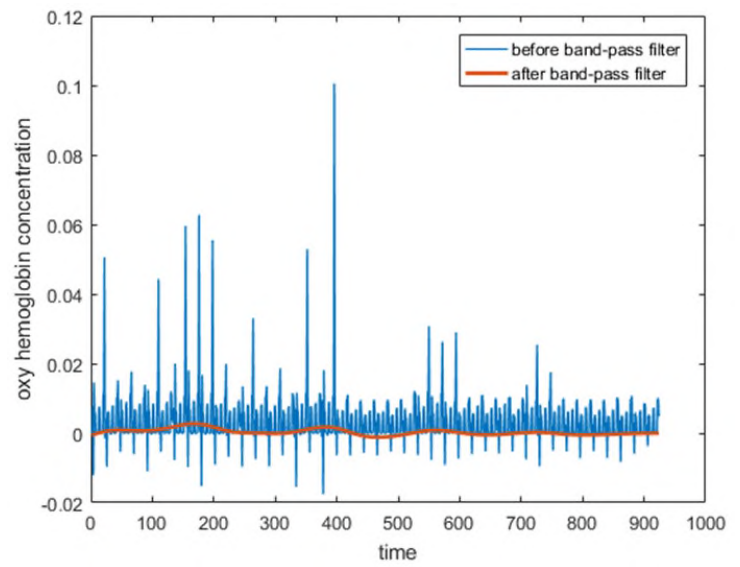

Fig. 4. Oxy hemoglobin concentration before and after using the bandpass filter when the first subject viewing lover's photo

We adopt GLM and CBNA methods for preprocessed data to analyse brain activation regions and brain function connections. We discuss the respective methodologies, and present some initial analysis using these techniques below.

Feasibility analysis of using GLM: Fig.5 illustrates the considerable differences in the degree of brain activation when subjects are viewing different types of pictures. Different types of pictures represent different intimate relationships. Viewing the pictures of strangers mainly activates the mid-back and the upper back of the medial upper back and the small areas nearby. Viewing the pictures of friends, on the other hand, mainly activates the frontal and middle gyrus of the frontal cortex and its vicinity. While viewing the lover's pictures activates the medial frontal upper back and upper frontal gyrus, and results in a higher level of activation in the trigonometric frontal gyrus, gyrus frontalis medius, the middle temporal gyrus, and the infraorbital frontal gyrus.

Results presented in [16] indicated that SR has a positive predictive effect concerning passion. Under the conditions of high and low SR, there are differences in the activation patterns and degrees of brain activation when individuals are stimulated by passion. Based on this observation, we compare the brain activation patterns of different subjects at different SR levels. As shown in Fig. 6, the differences in brain regions when viewing lovers' pictures are reflected mainly in the upper sacral and the posterior gyrus. Under pictures of strangers impression, the high SR group has a higher activation level in most areas of the middle frontal gyrus composed to the low SR group. When viewing the friend's pictures, the high SR group has a higher activation level in the trigonometric frontal gyrus and the middle frontal gyrus.

Brain network analysis: In this paper, we analyse the global small world characteristics and the three local brain networks characteristics of Betweenness Centrality, Node Degree, and Nodal Efficiency. Betweenness centrality is a measure of the centrality of nodes in a graph, which shows the importance of a node in transferring information to other nodes. It is given 


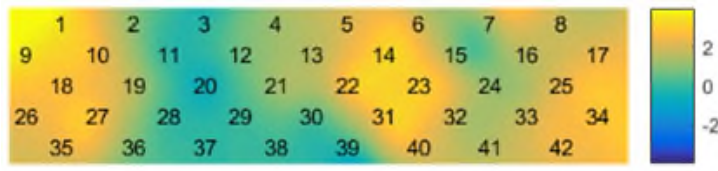

(a) Viewing Photos of Friends

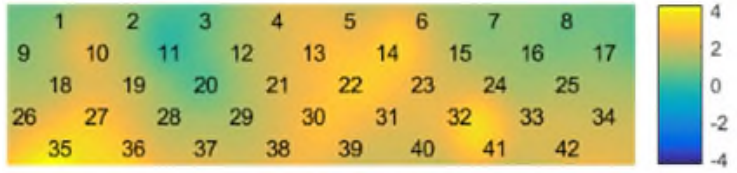

(b) Viewing Photos of Lovers

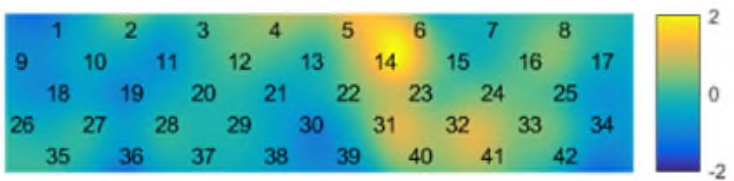

(c) Viewing Photos of Strangers

Fig. 5. The consistency t-value heat map of the brain activation area when viewing different pictures. Yellow represents a channel that is significantly activated, and blue represents a channel that is not significantly activated

as follows:

$$
b_{i}=\sum_{m \neq i \neq n \in G} \frac{\sigma_{m n}(i)}{\sigma_{m n}},
$$

where $\sigma_{m n}$ indicates the number of shortest path lengths from node $m$ to node $n$. Node degree is a basic measure of the connectivity between a node and other nodes in the network, which is defined as the number of edges of a node. It is defined as follows:

$$
K_{i}=\sum_{j \in G} a_{i j}
$$

where $a_{i j}$ indicates the value of the binary adjacency matrix.

Nodal Efficiency indicates the cost of exchanging energy in a network. It is defined as follows:

$$
e_{i}=\frac{1}{N-1} \sum_{j \neq i \in G} \frac{1}{d_{i j}},
$$

where $d_{i j}$ indicates the shortest path length between node $i$ and node $j$.

Fig. 7 (a) and (b) show the characteristic path length of different photos: friend < stranger < lover, and demonstrate that the difference between the clustering coefficients is not apparent when the sparsity is between 0.05 and 0.1 . This effect indicates that the brain has a higher overall efficiency in understanding the photos of friends, and there is a close relationship between the distant brain regions. This phenomenon may be due to increased information communication between the two brain regions when processing the friends' photos. Therefore, the characteristic path length of the functional network has decreased, and the overall efficiency has improved.

Fig.7(c) shows that the brain network of fNIRS signals can have distinct small world characteristics $(\sigma>1)$ when subjects are viewing different photos. The small world characteristics of the network indicate that the brain network is in an

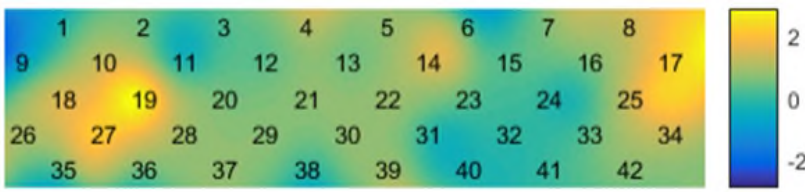

(a) Viewing Photos of Friends

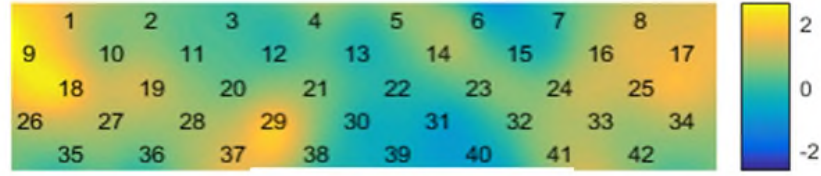

(b) Viewing Photos of Lovers

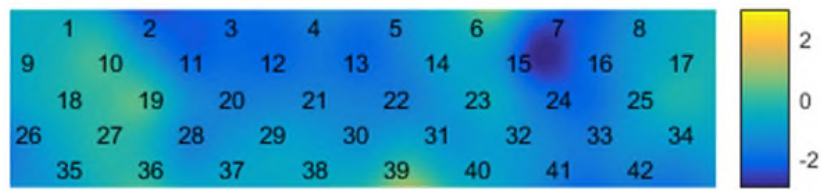

(c) Viewing Photos of Strangers

Fig. 6. The consistency t-value heat map of brain activation regions at different SR level when viewing different photos

efficient state of information transmission, which is conducive to the integration and processing of information. Since the characteristic path length of the viewing photos of the friends in the fNIRS brain network is smaller than that of the lovers and strangers, the clustering coefficients are not significantly different. According to the formula $\sigma=\gamma / \lambda>1$ (among them $\gamma \approx C_{p} / C_{p}^{\text {rand }}>1$ and $\lambda=L_{p} / L_{p}^{\text {rand }} \approx 1$, where $C_{p}$ and $C_{p}^{\text {rand }}$ represent clustering coefficients of brain network and random network, respectively, $L_{p}$ and $L_{p}^{\text {rand }}$ represent the characteristic path lengths of the brain network and the random network). The small world characteristics of viewing friend photos in the fNIRS brain network are larger than the others.

\section{Feature extraction}

In this experiment, the time domain features of the fNIRS signals were extracted from 42 channels across the full scalp. We adopted GLM and CBNA methods to extract useful features from the different intimate relationship groups. Statistical features from physiological signals were also extracted; these included the mean, median, max, min, and standard deviation (Std). The details of features from two signals are given in Table I.

The fNIRS and physiological features have different distributions. Therefore, it is necessary to normalise them for our multi-modal experiments. In this paper, we directly concatenated the feature matrices extracted from the two signals, and then normalise this fusion feature set using the $\mathrm{z}$-score method.

The $\mathrm{z}$-score method standardises data based on the mean and standard deviation of the raw data. The processed data conforms to the standard normal distribution; the mean is 0 , 


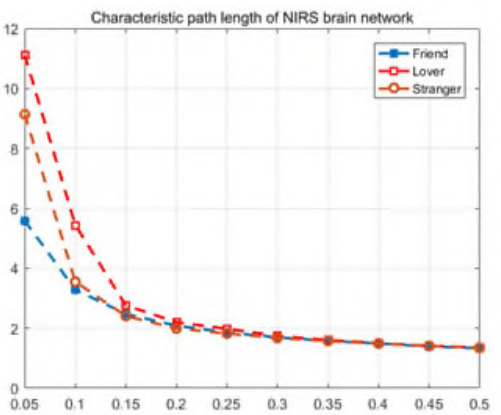

(a) Characteristic Path Length

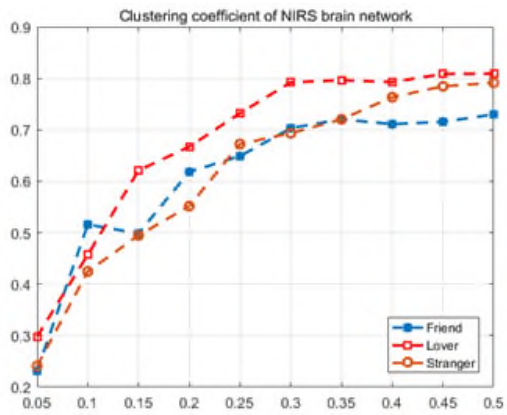

(b) Clustering Coefficients

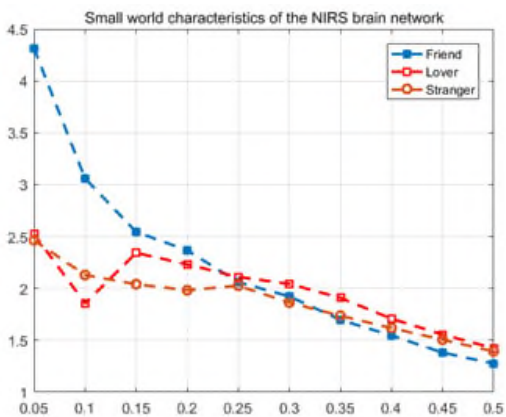

(c) Small World Characteristics

Fig. 7. The variation of the global network index of the fNIRS brain network with the sparsity when observing three different photos for all subjects. The abscissa indicates sparsity. (a) indicates the change of Characteristic Path Length of the three tasks with sparsity; (b) indicates the change of Clustering Coefficient of the three tasks with sparsity; (c) indicates the change of Small World Characteristic of the three tasks with sparsity.

TABLE I

ALL FEATURES OF FNIRS AND PHYSIOLOGICAL SIGNALS

\begin{tabular}{|l|l|l|l|l|l|l|}
\hline Signal & \multicolumn{5}{|c|}{ Type of features } \\
\hline \multirow{2}{*}{ fNIRS } & GLM & \multicolumn{4}{|c|}{ beta } \\
\cline { 2 - 7 } & CBNA & \multicolumn{2}{|c|}{ Betweenness Centrality } & Degree Centrality & \multicolumn{2}{|c|}{ Nodal Efficiency } \\
& & \multicolumn{2}{|c|}{ median } & max & min & std \\
\hline Physiological & Statistic & mean & max \\
\hline
\end{tabular}

and the standard deviation is 1 . The z-score function is:

$$
X^{*}=\frac{x-\mu}{\sigma}
$$

where $\mu$ denotes the mean of the original sample data, and $\sigma$ denotes the standard deviation of the original sample data.

\section{Feature selection}

In machine learning expirments, it is often necessary to collect a large number of information channels when analysing data, with these channels and their subsequent feature spaces containing a variety of characteristic variables. While this provides a rich source of information, these variables are often related and thus contain a certain amount of redundancy. Therefore, it can be desirable to construct new feature representations, which can hold as much of the useful information of the original data as possible while introducing mutual independence between the variarabes. In this regard, we tested several feature selection and reduction techniques in initial experiments, with LDA and PCA being identified as the most suitable for our needs.

We implemented PCA via singular value decomposition (SVD), which is based on a computational realisation function of a covariance matrix:

$$
\begin{gathered}
\varepsilon=\frac{1}{m} \sum_{i=1}^{n}\left(X^{(i)}\right)\left(X^{(i)}\right)^{T}, \\
C o v=\frac{X^{\prime} * X}{m} \\
{[U, S, V]=\operatorname{svd}(\operatorname{Cov}),}
\end{gathered}
$$

where $m$ represents the number of rows in 4 and $n$ represents the number of features. SVD was utilised to compute the eigenvectors and eigenvalues of the covariance matrix, noting $U$ denotes the eigenvector matrix, $S$ is the eigenvalues, $V$ is the weights, $S V D$ is the Matlab SVD application function, and $C o v$ is the Covariance matrix. The number of principal components was dynamically chosen in order to keep the retained variace at $85 \%$.

LDA is a linear dimensionality reduction algorithm [25]. Differing from PCA, LDA is supervised, it selects a projected hyperplane in $\mathrm{k}$-dimensional space such that the distance between projections of same classes on the hyperplane is as close as possible, while the distance between projections of different classes is as far as possible. The within-class scatter matrix as given by:

$$
S_{w}=\sum_{j=1}^{c} \sum_{i=1}^{N_{j}}\left(X_{i}^{j}-\mu_{j}\right)\left(X_{i}^{j}-\mu_{j}\right)^{T},
$$

where $X_{i}^{j}$ is the $i$-th sample of class $j, \mu_{j}$ is the mean of class $j, c$ is the number of classes, $N_{j}$ is the number of samples in class $j$. The between-class scatter matrix is then given by:

$$
S_{b}=\sum_{j=1}^{c}\left(\mu_{j}-\mu\right)\left(\mu_{j}-\mu\right)^{T},
$$

where $\mu$ represents the mean of all classes.

\section{E. Experimental setting}

In this paper, we ran tests on Support Vector Machines (SVM), LDA, k-nearest neighbours (KNN) and ensemble learning 
TABLE II

RESULTS OF FNIRS AND PHYSIOLOGICAL SIGNALS MULTI-MODEL CLASSIFICATION ACCURACY WITH LEAVE-ONE-SUBJECT-OUT CROSS-VALIDATION

\begin{tabular}{|l|c|c|c|c|}
\hline Features Classifier & SVM $(\%)$ & LDA $(\%)$ & KNN $(\%)$ & ensemble $(\%)$ \\
\hline Brain network+Statistic & 71.79 & 91.03 & 97.44 & $\mathbf{9 7 . 4 4}$ \\
\hline Beta+Statistic & 50.00 & 37.18 & 48.72 & $\mathbf{5 5 . 1 3}$ \\
\hline Brain network+Beta+Statistic & 56.41 & 39.74 & 91.03 & $\mathbf{9 8 . 7 2}$ \\
\hline
\end{tabular}

classifiers. In order to make full use of each sample's information, leave-one-subject-out cross-validation (LOSOCV) and 5 -fold cross-validation methods are used to validate our findings. The SVM uses a radial basis kernel function(sigma $=0.2, \mathrm{c}=3.5$ ).

TABLE III

RESULTS OF SINGLE MODEL CLASSIFICATION ACCURACY ON ENSEMBLE LEARNING

\begin{tabular}{|l|c|c|}
\hline Feature & 5-fold cross validation (\%) & $\begin{array}{l}\text { LOSOCV } \\
\text { (\%) }\end{array}$ \\
\hline Brain network & 97.33 & $\mathbf{9 7 . 4 4}$ \\
\hline Beta & 48.72 & $\mathbf{5 4 . 0 9}$ \\
\hline Brain network+Beta & 97.44 & $\mathbf{9 7 . 5 7}$ \\
\hline Statistic & 33.33 & $\mathbf{3 9 . 7 4}$ \\
\hline
\end{tabular}

\section{F. Experimental results and analysis}

The results of fNIRS and physiological signals, multimodal classification accuracy are shown in Table II. It can be seen that the ensemble learning performance is the strongest. Furthermore, the performance of LDA is slightly lower than other classifiers. Based on this observation, we adopt ensemble learning for the subsequent experiments.

The data in Table III shows the experimental results of the single model classification accuracy using ensemble learning. It can be observed that the brain network features of fNIRS signals are highly suitable for intimate relationship analysis. The results of utilising two feature extraction techniques, PCA and LDA are shown in Fig. 8. Comparing the two methods, the results of LDA are generally higher for different feature sets and improved on those achieved by PCA, which increase by $11.55 \%, 27.14 \%$ and $26.93 \%$ respectively.

We also analyse the effect of different SR level within our classification task. The results presented in Table IV indicate that higher classification rates are achieved with subjects with mean SR levels; this observation is consistent across the different feature sets. Experimental results also indicate that the accuracy of the multi-modal signal is slightly higher than the uni-modal systems.

\section{CONCLUSION}

Through multi-modal experiment utilising fNIRS signals and physiological signals, we found evidence that different brain activation regions and patterns are produced when a person is viewing different types of photos showing different forms of

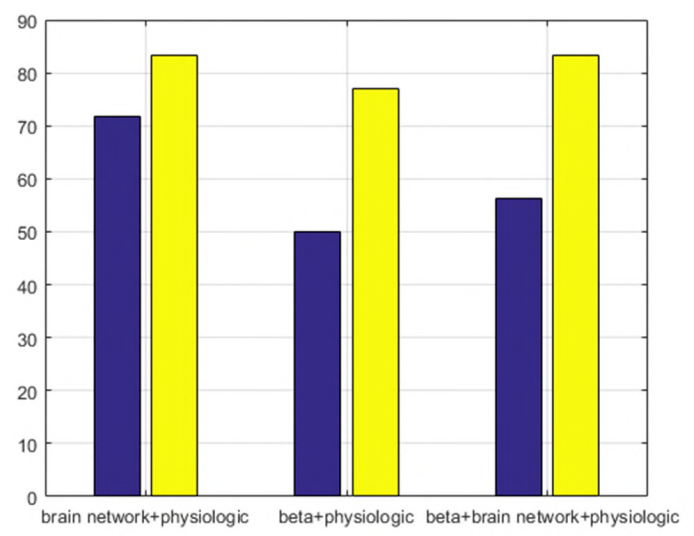

Fig. 8. Comparison of the results of the bimodal feature set using different feature selection methods on the SVM classifier. The blue bar indicates PCA, and the yellow bar indicates LDA

intimate relationships. We found that we could also predict the different activation states using machine- and deep-learning methods. We observed that brain network features extracted from fNIRS signals achieve higher classification accuracy than different modalities across a range of different classifiers.

At present, the studies into intimate relationships have mainly focused on close relationships such as marriage and love, or the relationship between adult attachment and other psychological traits. However, this approach is not systematic, nor mature. Therefore, we plan to extend our research into more real-world settings such that the findings are adaptable into people's real life, such as in psychological counselling, or the treatment of family marital relationships. A good understanding of the relationships can also help people to strengthen their ability to maintain intimate relationships, thereby promoting the harmony of intimate relationships, family and social harmony.

\section{REFERENCES}

[1] E. Kirchler, C. Rodler, E. Holzl, and K. Meier, Conflict and decision making in close relationships: Love, money and daily routines. Psychology Press, 2013.

[2] A. Bartels and S. Zeki, "The neural basis of romantic love," Neuroreport, vol. 11 , no. 17 , pp. 3829-3834, 2000.

[3] B. P. Acevedo, A. Aron, H. E. Fisher, and L. L. Brown, "Neural correlates of long-term intense romantic love," Social cognitive and affective neuroscience, vol. 7, no. 2, pp. 145-159, 2012.

[4] S. K. Piper, A. Krueger, S. P. Koch, J. Mehnert, C. Habermehl, J. Steinbrink, H. Obrig, and C. H. Schmitz, "A wearable multi-channel fnirs system for brain imaging in freely moving subjects," Neuroimage, vol. 85 , pp. 64-71, 2014 
TABLE IV

RESULTS OF DIFFERENT SR LEVEL ON ENSEMBLE LEARNING CLASSIFICATION ACCURACY

\begin{tabular}{|l|l|l|l|l|l|l|}
\hline SR level Types of feature & $\begin{array}{l}\text { Brain } \\
\text { network(\%) }\end{array}$ & Beta(\%) & $\begin{array}{l}\text { Brain net- } \\
\text { work+Beta(\%) }\end{array}$ & Statistic+Beta(\%) & $\begin{array}{l}\text { Statistic+Brain } \\
\text { network(\%) }\end{array}$ & $\begin{array}{l}\text { Statistic+Beta+Brain } \\
\text { network(\%) }\end{array}$ \\
\hline High SR & 93.33 & 36.67 & 96.67 & 40.00 & 96.67 & 96.67 \\
\hline Low SR & 91.67 & 33.33 & 66.67 & 33.33 & 66.67 & 91.67 \\
\hline Mean SR & $\mathbf{9 7 . 4 4}$ & $\mathbf{5 4 . 0 9}$ & $\mathbf{9 7 . 5 7}$ & $\mathbf{5 5 . 1 3}$ & $\mathbf{9 7 . 4 4}$ & $\mathbf{9 8 . 7 2}$ \\
\hline
\end{tabular}

[5] M. R. Bhutta, M. J. Hong, Y.-H. Kim, and K.-S. Hong, "Single-trial lie detection using a combined fnirs-polygraph system," Frontiers in psychology, vol. 6, pp. 709:1-9, 2015.

[6] A. Neupane, N. Saxena, and L. Hirshfield, "Neural underpinnings of website legitimacy and familiarity detection: An fnirs study," in Proceedings of the 26th International Conference on World Wide Web, ser. WWW'17, Republic and Canton of Geneva, Switzerland, 2017, pp. $1571-1580$.

[7] I. Kovelman, M. H. Shalinsky, M. S. Berens, and L.-A. Petitto, "Shining new light on the brain's "bilingual signature": a functional near infrared spectroscopy investigation of semantic processing," Neuroimage, vol. 39, no. 3, pp. 1457-1471, 2008.

[8] C. Herff, D. Heger, F. Putze, C. Guan, and T. Schultz, "Cross-subject classification of speaking modes using fnirs," in Neural Information Processing. ICONIP 2012. Berlin, Heidelberg: Springer Berlin Heidelberg, 2012, pp. 417-424.

[9] G. Aranyi, F. Pecune, F. Charles, C. Pelachaud, and M. Cavazza, "Affective interaction with a virtual character through an fnirs braincomputer interface," Frontiers in computational neuroscience, vol. 10, pp. 70:1-14, 2016.

[10] G. Aranyi, F. Charles, and M. Cavazza, "Anger-based bci using fnirs neurofeedback," in Proceedings of the 28th Annual ACM Symposium on User Interface Software \& Technology, ser. UIST '15. New York, NY, USA: ACM, 2015, pp. 511-521.

[11] D. A. Boas, C. E. Elwell, M. Ferrari, and G. Taga, "Twenty years of functional near-infrared spectroscopy: introduction for the special issue," Elsevier, 2014.

[12] M. Ferrari and V. Quaresima, "A brief review on the history of human functional near-infrared spectroscopy (fnirs) development and fields of application," Neuroimage, vol. 63, no. 2, pp. 921-935, 2012.

[13] M. Dan, A. Saha, A. Konar, A. L. Ralescu, and A. K. Nagar, "A type-2 fuzzy approach towards cognitive load detection using fnirs signals," in 2016 IEEE International Conference on Fuzzy Systems (FUZZ-IEEE), July 2016, pp. 2508-2515.

[14] S. Chen, Z. Gao, and S. Wang, "Emotion recognition from peripheral physiological signals enhanced by eeg," in 2016 IEEE International
Conference on Acoustics, Speech and Signal Processing (ICASSP), March 2016, pp. 2827-2831.

[15] K. Veselkov and B. Schuller, "The age of data analytics: converting biomedical data into actionable insights," Methods, vol. 151, pp. $1-2$ 2018.

[16] R. Yang, "The mechanism of strengthening sensitivity in college students'love passion," Master's thesis, Tianjin Normal University, 2017.

[17] P. J. Corr, "Testing problems in ja gray's personality theory: a commentary on," Personality and Individual Differences, vol. 30, no. 2, pp. 333-352, 2001

[18] M. L. Schroeter, M. M. Bcheler, M. Karsten, U. Kamil, O. Hellmuth, L. Gabriele, T. Marc, V. Arno, and V. C. D Yves, "Towards a standard analysis for functional near-infrared imaging," Neuroimage, vol. 21, no. 1, pp. 283-290, 2004.

[19] X. P. Ding, G. Fu, and K. Lee, "Neural correlates of own-and otherrace face recognition in children: a functional near-infrared spectroscopy study," Neuroimage, vol. 85, pp. 335-344, 2014.

[20] S. Tak and J. C. Ye, "Statistical analysis of fnirs data: a comprehensive review," Neuroimage, vol. 85, pp. 72-91, 2014.

[21] P. Pinti, A. Merla, C. Aichelburg, F. Lind, S. Power, E. Swingler, A. Hamilton, S. Gilbert, P. W. Burgess, and I. Tachtsidis, "A novel glm-based method for the automatic identification of functional events (aide) in fnirs data recorded in naturalistic environments," NeuroImage, vol. 155, pp. 291-304, 2017.

[22] K. J. Friston, "Functional and effective connectivity: a review," Brain connectivity, vol. 1, no. 1, pp. 13-36, 2011.

[23] B. S. Khundrakpam, A. Reid, J. Brauer, F. Carbonell, J. Lewis, S. Ameis, S. Karama, J. Lee, Z. Chen, S. Das et al., "Developmental changes in organization of structural brain networks," Cerebral Cortex, vol. 23, no. 9, pp. 2072-2085, 2012.

[24] M. Rubinov, S. A. Knock, C. J. Stam, S. Micheloyannis, A. W. Harris, L. M. Williams, and M. Breakspear, "Small-world properties of nonlinear brain activity in schizophrenia," Human brain mapping, vol. 30, no. 2, pp. 403-416, 2009.

[25] A. M. Martínez and A. C. Kak, "Pca versus 1da," IEEE transactions on pattern analysis and machine intelligence, vol. 23, no. 2, pp. 228-233, 2001. 